\title{
BET bromodomain inhibitor JQ1 modulates microRNA expression in thyroid cancer cells
}

\author{
CATIA MIO $^{1}$, KETTY CONZATTI $^{1}$, FEDERICA BALDAN $^{2}$, LORENZO ALLEGRI $^{1}$, \\ MARIALUISA SPONZIELLO ${ }^{2}$, FRANCESCA ROSIGNOLO ${ }^{2}$, DIEGO RUSSO $^{3}$, \\ SEBASTIANO FILETTI $^{2}$ and GIUSEPPE DAMANTE ${ }^{1,4}$
}

\author{
${ }^{1}$ Department of Medical Area, University of Udine, I-33100 Udine; ${ }^{2}$ Department of Internal Medicine \\ and Medical Specialties, University 'Sapienza', I-00185 Rome; ${ }^{3}$ Department Health Sciences, \\ University of Catanzaro 'Magna Graecia', I-88100 Catanzaro; ${ }^{4}$ Institute of Medical Genetics, \\ University Hospital 'S. Maria della Misericordia', I-33100 Udine, Italy
}

Received May 11,2017; Accepted October 10, 2017

DOI: $10.3892 /$ or.2017.6152

\begin{abstract}
Anaplastic thyroid carcinoma (ATC) represents the most lethal thyroid cancer sub-type, currently unresponsive to standard treatments. Recently, bromodomain and extra-terminal (BET) proteins have emerged as attractive therapeutic targets in several diseases, including cancer. In different cancer models, the anti-neoplastic activity of BET inhibitors such as JQ1, I-BET762 and I-BET151 have already been established, due to both direct and indirect effects. miRNAs are 20-22 nucleotide transcriptional regulators which play important roles in proliferation, differentiation and apoptosis. Hitherto, the relationship between JQ1 and miRNAs has not been explored. The goal of this study was to delineate JQ1-associated miRNA regulation in ATC cells. Two ATC-derived cell lines (SW1736 and 8505c) were treated with either $5 \mu \mathrm{M}$ JQ1 or vehicle for 48 or $72 \mathrm{~h}$. A non-tumorigenic thyroid cell line (Nthy-ori 3-1) was used as a control. miRNome analysis displayed a JQ1-related dysregulation of several miRNAs, 7 of which turned out to be commonly dysregulated in both cell lines at both time-points. Furthermore, miR-4516 turned out to be downregulated in both ATC cell lines, when compared to the non-tumorigenic
\end{abstract}

Correspondence to: Professor Giuseppe Damante, Department of Medical Area, University of Udine, Piazzale Kolbe 1, I-33100 Udine, Italy

E-mail: giuseppe.damante@uniud.it

Abbreviations: ATC, anaplastic thyroid cancer; BET, bromodomain and extra-terminal protein; DMSO, dimethyl sulphoxide; miRNA, microRNA; PDTC, poorly differentiated thyroid cancer; RNA-seq, RNA sequencing; STAT, signal transducer and activator of transcription; STR, single tandem repeats; UTR, untranslated region; WDTC, well-differentiated thyroid cancer

Key words: JQ1, miRNA, nCounter, anaplastic thyroid cancer, miR-4516 ones and notably, JQ1 treatment in both ATC cell lines induced its upregulation, restoring, in some way, its basal expression levels. We, therefore, focused on miR-4516 expression and STAT3 levels, since it was previously predicted to be a putative target of this microRNA. Consistently, phospho-STAT3 and its target $\mathrm{p} 21^{\text {Waf1/Cip1 }}$ turned out to be downregulated and upregulated in both JQ1-treated ATC cell lines, respectively. Thus, our data revealed that modulation of miRNA expression is one of the multiple mechanisms of the effect of JQ1 in thyroid cancer cells.

\section{Introduction}

Thyroid cancer (TC) is the most common malignancy of the endocrine system and is classified as well differentiated (WDTC), poorly differentiated (PDTC) and anaplastic thyroid carcinoma (ATC) $(1,2)$. ATC, which is composed of undifferentiated cells arising from thyroid follicular epithelium, represents the least common but the most lethal thyroid cancer sub-type (3), since it is unresponsive to standard treatments.

An innovative approach employs compounds that target chromatin readers and, so far, BET proteins are the best characterized ones. BET is the acronym for bromodomain and extra-terminal domain family of proteins (4) that act as acetyllysine readers, which are known to be associated with the transfer of epigenetic information by the so-called 'write-readerase' concept (5). BET inhibitors (BETi) have recently attained the consensus of researchers as well established antineoplastic agents in different cancer models (3,6-8), and JQ1, I-BET762 and I-BET151 are the better characterized drugs (9). A consensus in epigenetic drug mechanisms of action is that their anti-neoplastic activity is due both by direct and indirect effects. We have already established that BET inhibition impairs different key biological pathways in human ATC cell lines, deeming these epigenetic drugs as promising therapeutic tools for thyroid cancer (3).

MicroRNAs (miRNAs) are 20-22 nucleotide endogenous non-coding RNA molecules that regulate transcription and/or translation by controlling the expression of a large number of genes, binding to specific sites (called seeds) in the target 
mRNA, i.e., 3'UTR (untranslated region). This interaction is generally involved in mRNA cleavage/degradation or translational suppression (1). Therefore, these small molecules play important roles in development, metabolism, proliferation, differentiation and apoptosis. Consequently, due to their leading role, miRNAs are promising therapeutic targets in many diseases, including thyroid cancer $(1,10)$. Several miRNAs were found to be dysregulated in thyroid cancer $(11,12)$. For these many reasons, the aim of our study was to demonstrate a link between JQ1 treatment and miRNA regulation in thyroid cancer.

\section{Materials and methods}

Human cell lines. SW1736 (obtained from Cell Lines Service $\mathrm{GmbH}$, Eppelheim, Germany) and 8505c (purchased from Sigma-Aldrich, St. Louis, MO, USA) are human cell lines derived from ATC $(13,14)$, while Nthy-ori 3-1 (purchased from Sigma-Aldrich) is a human thyroid follicular epithelial cell line immortalized by the SV40 large T gene. Cell lines were tested and confirmed to be mycoplasma-free and authenticated by STR analysis as appropriate cell lines of thyroid cancer origin. Cell lines were grown in RPMI-1640 medium (EuroClone S.p.A, Pero MI, Italy) supplemented with $10 \%$ fetal bovine serum (Gibco Invitrogen, Milan, Italy) and $50 \mathrm{mg} / \mathrm{ml}$ gentamicin (Gibco Invitrogen), in a humidified incubator $\left(5 \% \mathrm{CO}_{2}\right.$ in air at $37^{\circ} \mathrm{C}$ ) (Eppendorf AG, Hamburg, Germany). Cultured cells were treated with vehicle (DMSO, Sigma-Aldrich) or JQ1 (5 $\mu \mathrm{M}$ in DMSO) (Cayman Chemical, Ann Arbor, MI, USA) for either 48 or $72 \mathrm{~h}$.

miRNA expression and normalization. Expression analysis of 812 miRNAs was performed by the genetic laboratory of Pharmadiagen Srl, using the NanoString nCounter v2 miRNA Assay kit (NanoString Technologies, Seattle, WA, USA). The nCounter miRNA expression assay was used to detect and count miRNAs through hybridization with fluorescentlylabeled barcoded probes. A total of $280 \mathrm{ng}$ of RNA was processed according to the manufacturer's instructions (NanoString Technologies), including preparation and the hybridization protocol performed at $65^{\circ} \mathrm{C}$ for $16 \mathrm{~h}$. Subsequent purification and detection of miRNA target were carried out using the nCounter Analysis system.

The total miRNA counts obtained were elaborated and normalized following the Nanostring's Data Analysis Guide. The background correction was calculated as the geometric mean of negative control plus $2 \mathrm{X}$ standard deviation and subtracted to each count. miRNAs expressing less than the background were setting as not expressed to overcome basal noise. The technical normalization was performed using the positive control spike counts. We calculated the normalization factor as the ratio between the average of geometric means and the geometric mean of each sample. The counts of every miRNAs were multiply by the normalization factor. To be more conservative and avoid false positive miRNA, we set an additional cut off (15 counts) after the above normalization. Fold change was, then, calculated as JQ1-treated vs. vehicle or ATC cell line vs. NThy ori 3.1. Only miRNA showing a deregulation greater than two $\log 2$-fold change were taken into consideration.

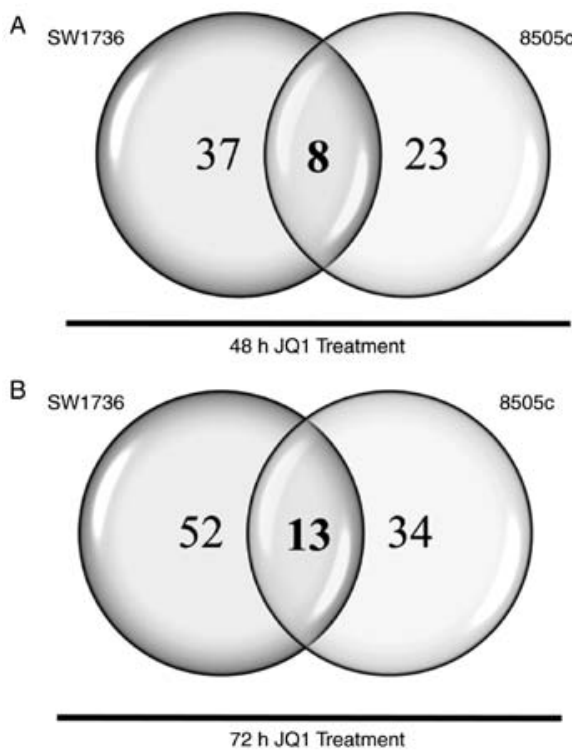

Figure 1. miRNA alteration due to JQ1 treatment in ATC cells. (A) SW1736 and $8505 \mathrm{c}$ cells treated with $5 \mu \mathrm{M}$ JQ1 or vehicle for $48 \mathrm{~h}$ were subjected to NanoString platform miRNA evaluation. A total of 45 and 31 miRNAs turned out to be upregulated by JQ1 in SW1736 and 8505c, respectively. Eight miRNAs shared a common alteration in both cell lines. (B) SW1736 and $8505 \mathrm{c}$ treated with $5 \mu \mathrm{M} \mathrm{JQ} 1$ or vehicle for $72 \mathrm{~h}$ were subjected to NanoString platform miRNA evaluation. A total of 65 and 47 miRNAs turned out to be upregulated by BET inhibition in SW1736 and 8505c, respectively. Thirteen miRNAs shared a common alteration in both cell lines.

MicroRNA expression validation. Briefly, $200 \mathrm{ng}$ of total RNA, treated with either JQ1 $5 \mu \mathrm{M}$ or vehicle, was extracted and reverse-transcribed using miRCURY LNA ${ }^{\mathrm{TM}}$ Universal cDNA Synthesis kit II (Exiqon, Vedbaek, Denmark) as described by the manufacturer. Before reverse transcription, 108 copies of UniSP6 synthetic spike-in were added to each condition, as a quality control for subsequent analyses. Real-time PCRs were performed using ExiLENT SYBR ${ }^{\circledR}$ Green master mix (Exiqon) with the ABI Prism 7300 Sequence Detection System (Applied Biosystems, Foster City, CA, USA). SNORD44 was used as an inter-plate calibrator. The $\Delta \Delta \mathrm{CT}$ method, by means of the SDS software (Applied Biosystems), was used to calculate microRNA levels. All oligonucleotide primers were purchased from Exiqon. Each sample was run in triplicate and experiments were repeated at least three times using at least two independent samples.

Protein extraction and western blotting. Total protein extraction was performed as previously described (15). Briefly, SW1736 and 8505c cells, incubated in vehicle-treated medium (NT, untreated cultures) or with JQ1 $5 \mu \mathrm{M}$, were harvested by scraping and lysed with total lysis buffer (Tris-HCl $50 \mathrm{mM}$ $\mathrm{pH} 8.0, \mathrm{NaCl} 120 \mathrm{mM}$, EDTA $5 \mathrm{mM}$, Triton 1\%, NP40 1\%, protease inhibitors). For western blot analysis, the proteins were electrophoresed on $10 \%$ SDS-PAGE and then transferred to nitrocellulose membranes, which were saturated with $5 \%$ non-fat dry milk in PBS/0.1\% Tween-20. The membranes were then incubated overnight with rabbit polyclonal anti-p21 antibody 1:500 (Santa Cruz Biotechnology, Inc., Heidelberg, Germany), rabbit polyclonal anti-p27 antibody 1:500 (Santa Cruz Biotechnology, Inc.), rabbit monoclonal anti-phoshpo-STAT3 (Tyr705) antibody 1:10,000 [Merck 

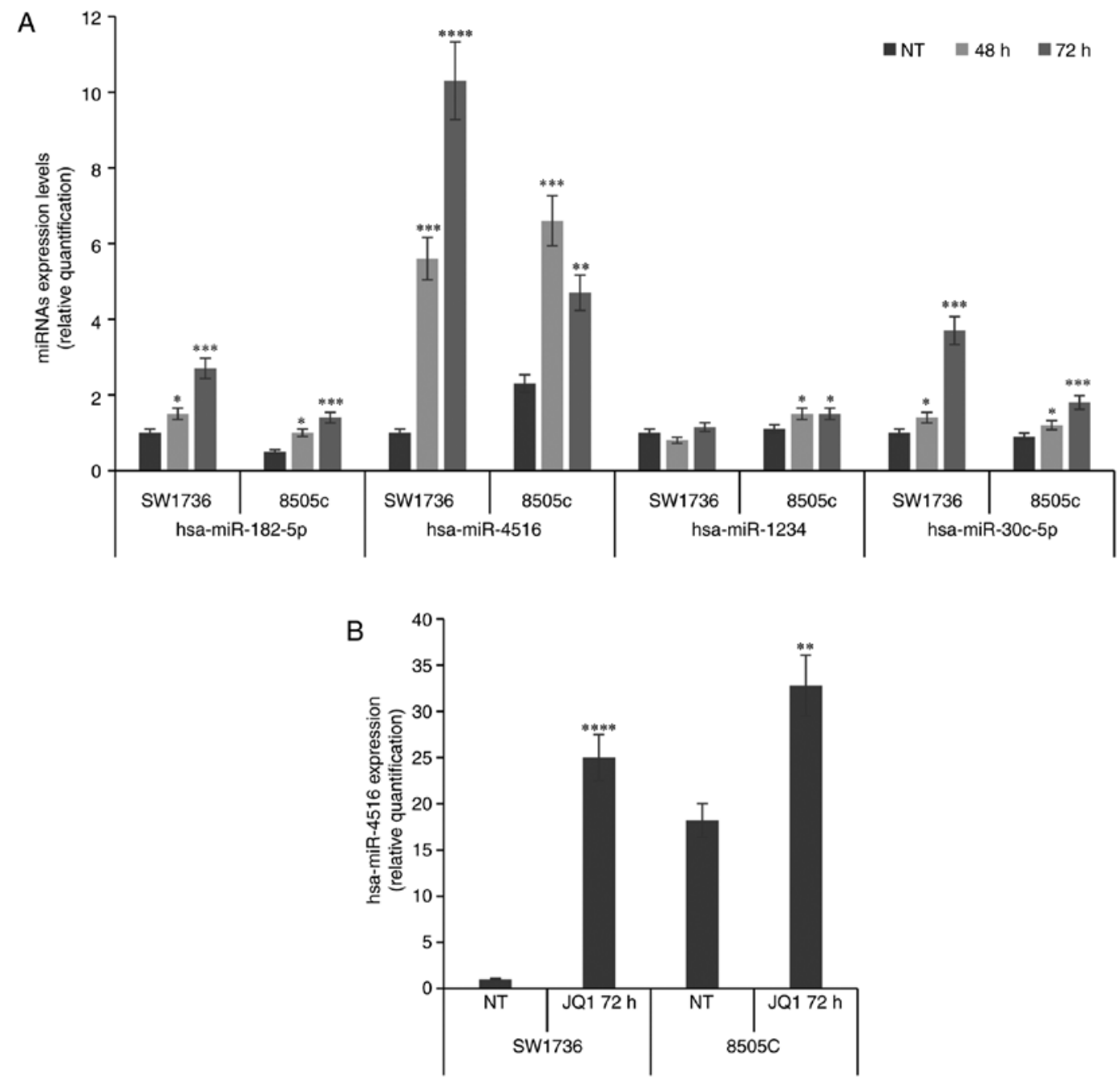

Figure 2. miRNome data validation. (A) MicroRNA expression levels identified by nCounter analysis were confirmed by qPCR using on-demand Exiqon primers. (B) miR-4516 expression validation in biological replicates. All data were normalized to SNORD44 levels. Vehicle-treated (NT) SW1736 cells were arbitrarily set at 1.0 and miRNA levels are expressed as the relative expression values. Results are shown as the mean $\pm \mathrm{SD} .{ }^{*} \mathrm{P}<0.5,{ }^{* *} \mathrm{P}<0.01,{ }^{* * *} \mathrm{P}<0.001$, ${ }_{* * * * * *}^{*}<0.0001$ by ANOVA test.

Millipore, Vimodrone (MI), Italy], mouse monoclonal anti-STAT3 antibody 1:500 (Santa Cruz Biotechnology, Inc.) or rabbit anti-actin antibody 1:1,000 (Abcam, Cambridge, UK). The following day, the membranes were incubated for $2 \mathrm{~h}$ with anti-rabbit immunoglobulin coupled to peroxidase 1:4,000 (Sigma-Aldrich). The blots were developed using UVItec Alliance LD (UVItec Ltd., Cambridge, UK) with SuperSignal Technology (Thermo Fisher Scientific Inc., Waltham, MA, USA).

Statistical analysis. MicroRNA and protein levels were expressed as the means \pm SD, and significances were determined using a one-way ANOVA followed by Dunnett's test performed with GraphPad Software for Science (San Diego, CA, USA). P-value $<0.05$ was considered to indicate a statistically significant difference.

\section{Results}

JQ1 treatment modifies miRNA expression in ATC. Our previously published data demonstrated the anti-neoplastic activity of $5 \mu \mathrm{M} \mathrm{JQ1}$ in anaplastic thyroid cancer cells as indicated by a decrease in cell viability coupled by an increase in cell death phenomena and cell cycle arrest (3). Moreover, our RNA-seq analysis revealed the complexity of the JQ1 mechanism of action, which takes advantage of hundreds of direct and indirect targets.

To elucidate the possible role of miRNAs in ATC therapeutic response after JQ1 treatment, we used the nCounter miRNA Expression Assay kit, a novel digital color-coded barcode technology based on direct multiplexed assessment of gene expression. Two ATC cell lines, SW1736 and 8505c, JQ1- or vehicle-treated for either 48 or $72 \mathrm{~h}$, were run on the NanoString platform. We chose these time-points as it was demonstrated that miRNA half-lives usually exceeded $24 \mathrm{~h}$ (16). Of the 812 miRNAs analyzed, 45 and 31 miRNAs turned out to be dysregulated after $48 \mathrm{~h}$ of JQ1 treatment in SW1736 and 8505c cells, respectively. As shown in Fig. 1A, 8 miRNAs displayed a common upregulation in both cell lines. After $72 \mathrm{~h}$ of JQ1 treatment, conversely, 65 and 47 miRNAs were demonstrated to be dysregulated in SW1736 and 8505c cells, respectively. Thirteen miRNAs exhibited a common upregulation in the two cell lines (Fig. 1B). In order to delineate central miRNAs in JQ1-mediated effects, we compared common miRNAs at 
Table I. Shared miRNAs altered after both 48 and $72 \mathrm{~h}$ of JQ1 $5-\mu \mathrm{M}$ treatment in ATC cells.

\begin{tabular}{llll}
\hline & & \multicolumn{2}{c}{ Fold change } \\
\cline { 3 - 4 } miRNAs & Cells & $48 \mathrm{~h}$ & $72 \mathrm{~h}$ \\
\hline hsa-miR-182-5p & SW1736 & 2.97 & 4.07 \\
& $8505 \mathrm{c}$ & 7.99 & 9.34 \\
hsa-miR-4516 & SW1736 & 3.03 & 3.58 \\
& $8505 \mathrm{c}$ & 3.44 & 3.41 \\
hsa-miR-1234 & SW1736 & 4.26 & 3.82 \\
& $8505 \mathrm{c}$ & 2.75 & 2.26 \\
hsa-miR-30c-5p & $\mathrm{SW1736}$ & 4.50 & 5.26 \\
& $8505 \mathrm{c}$ & 7.70 & 8.15 \\
hsa-miR-4488 & $\mathrm{SW1736}$ & 9.39 & 9.58 \\
& $8505 \mathrm{c}$ & 4.30 & 4.32 \\
hsa-miR-4532 & $\mathrm{SW1736}$ & 9.01 & 9.10 \\
& $8505 \mathrm{c}$ & 8.96 & 9.73 \\
hsa-miR-548t-5p & $\mathrm{SW1736}$ & 7.54 & 7.78 \\
& $8505 \mathrm{c}$ & 2.83 & 3.21 \\
\hline
\end{tabular}

48 and $72 \mathrm{~h}$, highlighting a pool of 7 miRNAs commonly upregulated in both SW1736 and 8505c after both 48 and $72 \mathrm{~h}$ of JQ1 treatment (Table I).

To validate the miRNA expression data, we performed qPCR analysis of these seven differentially expressed miRNAs. Out of the seven microRNAs, significant changes were observed only for three miRNAs (miR-182-5p, miR$30 c-5 p$ and miR-4516), as shown in Fig. 2A. Notably, validation of miR-4488/4532/548t-5p was not possible due to technical issues. Among the validated microRNAs, after further validation in biological replicates in which a consistent increase in miR-4516 levels after JQ1 treatment was demonstrated (Fig. 2B), we focused our attention on miR-4516 and its downstream pathway, since this microRNA was previously linked to apoptotic phenomena (17). JQ1 treatment increased both apoptotic and necrotic phenomena (represented by caspase-3/7 activity levels and PARP to cleaved-PARP ratio) in both ATC cell lines, as demonstrated in our previously published study (3).

JQ1 effects on miR-4516 levels and STAT3-dependent pathway. We exploited the target prediction database miRDB (http://mirdb.org/miRDB/) to delineate miR-4516 potential mRNA targets. Of the 1545 hits, the signal transducer and activator of transcription 3 (STAT3) was already characterized as a miR-4516 downstream effector. STAT3 is a transcription factor that, when phosphorylated, relocates into the nucleus where it acts as a transcription activator. Besides its normal functions, this protein plays an important role in cellular transformation and tumorigenesis and it was revealed to be overexpressed and hyper-activated in thyroid cancer (18-20). Once activated, STAT3 induces the expression of SKP2, a member of the F-box protein family, an oncogene that mediates the ubiquitination of p21 (CDKN1A)
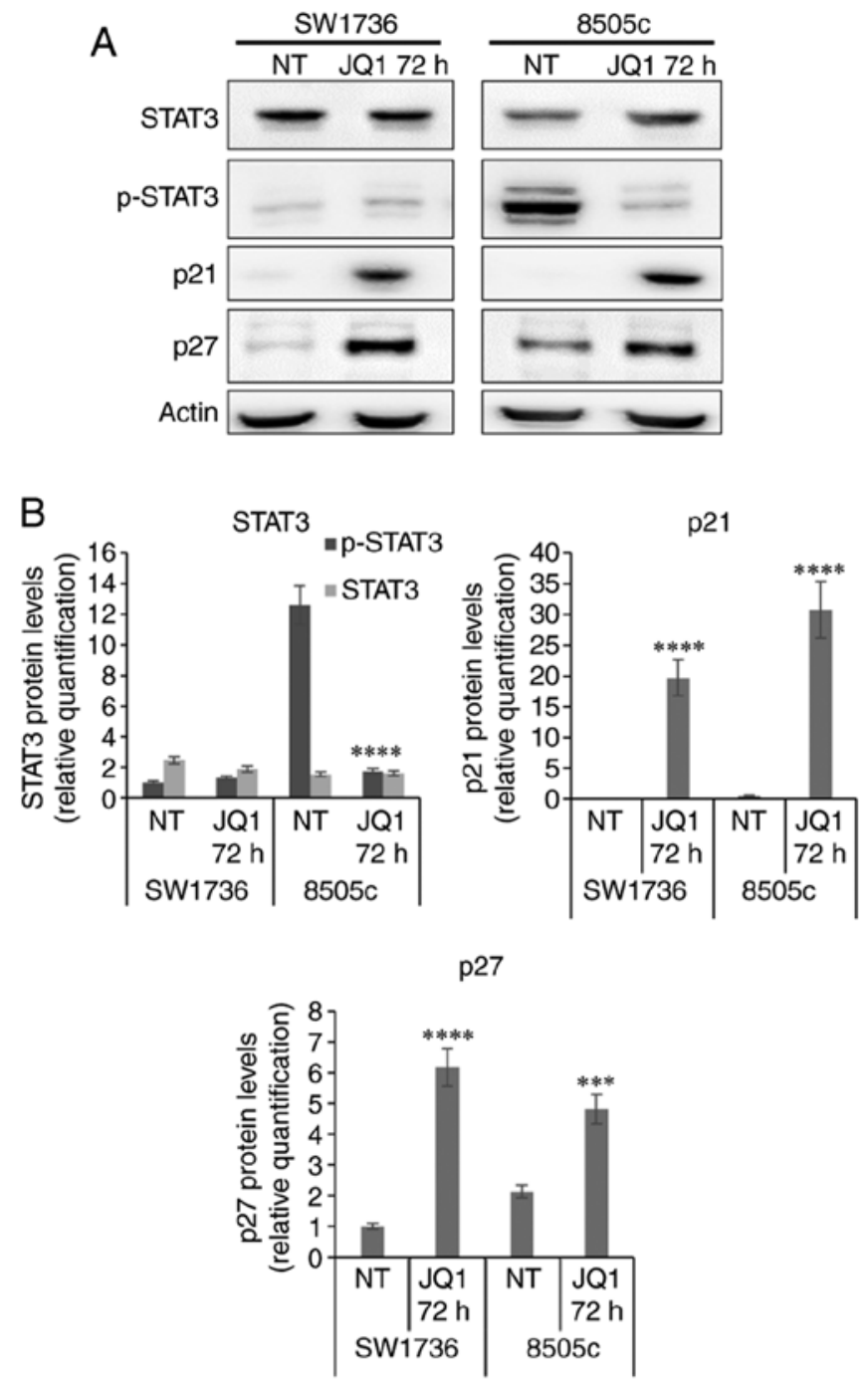

Figure 3. JQ1-dependent STAT3 pathway regulation in ATC cells. (A) SW1736 and $8505 \mathrm{c}$ were treated with $5 \mu \mathrm{M}$ JQ1 or vehicle for $72 \mathrm{~h}$. The cells were collected and STAT3, p-STAT3 (Tyr705), p21 and p27 protein levels were evaluated. (B) Densitometric analysis of STAT3, p-STAT3, p21 and p27 protein levels in ATC cells after $5 \mu \mathrm{M}$ JQ1 or vehicle treatment. Vehicle-treated (NT) SW1736 cells were arbitrarily set at 1.0 and protein levels were expressed as the relative expression value. The results are displayed as the mean $\pm \mathrm{SD} .{ }^{* * * *} \mathrm{P}<0.001,{ }^{* * * *} \mathrm{P}<0.0001$ by ANOVA test.

and p27 (CDKN1B) decreasing the tumorigenic potential of cells $(21,22)$.

Cell line $8505 \mathrm{c}$ treated with $5 \mu \mathrm{M} \mathrm{JQ1}$ displayed a strong decrease in phospho-STAT3 (p-STAT3) protein levels when compared to the vehicle-treated cells. SW1736, conversely, did not display any detectable variation in p-STAT3 protein levels after treatment; this was possibly due to a diverse site of phosphorylation (i.e., Ser727), different from the one detected with our antibody (Tyr705) (Fig. 3A and B). Moreover, Sos et al reported a delayed STAT3 activation by Tyr705 phosphorylation in SW1736 cells, hypothesizing an IL6-related autocrine loop that may be responsible for the time-dependent activation of this signaling pathway (23). We, then, evaluated whether a reduction in p-STAT3 corresponded in a downregulation of SKP2 expression. After both $48 \mathrm{~h}$ and $72 \mathrm{~h}$ of JQ1 treatment, ATC cells displayed a significant decrease in SKP2 mRNA levels (Fig. 4), corrobo- 


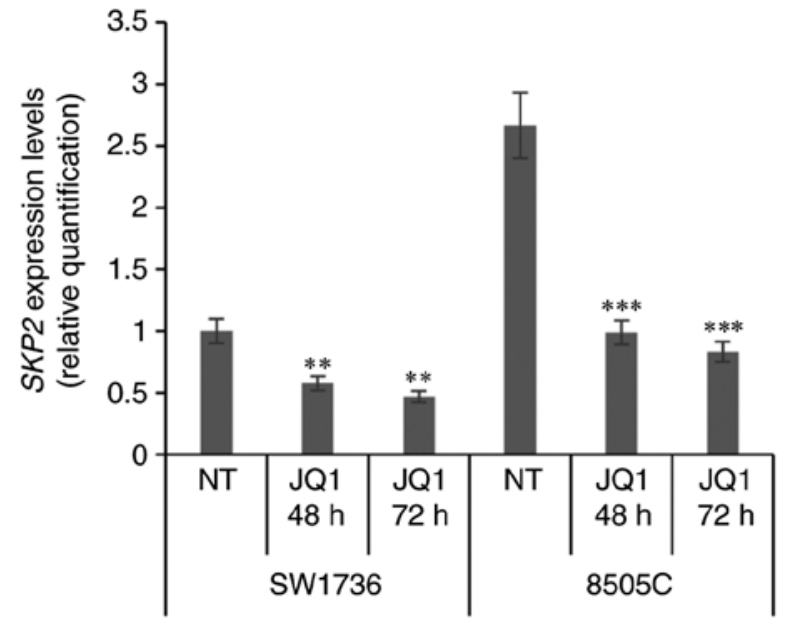

Figure 4. JQ1-related SKP2 regulation in ATC cells. SW1736 and 8505c were treated with $5 \mu \mathrm{M}$ JQ1 or vehicle for 48 and $72 \mathrm{~h}$. The cells were collected and SKP2 mRNA levels were evaluated. Vehicle-treated (NT) SW1736 cells were arbitrarily set at 1.0 and the mRNA levels were expressed as the relative expression values. Results are displayed as the mean $\pm \mathrm{SD} .{ }^{* *} \mathrm{P}<0.01$, ${ }^{* * * *} \mathrm{P}<0.001$ by ANOVA test.

A

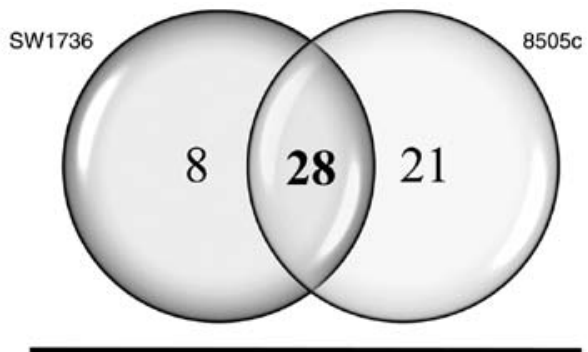

Upregulated in ATC

B

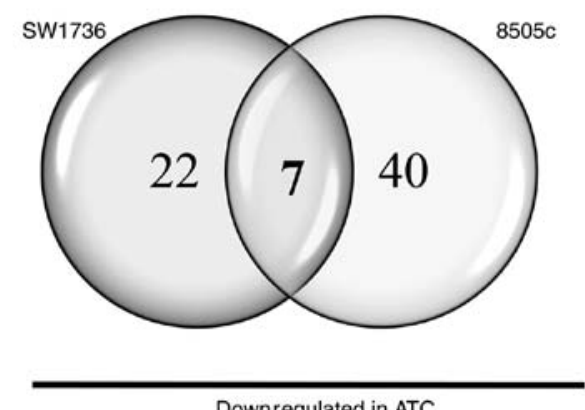

Downregulated in ATC

Figure 5. miRNA alteration in ATC cells compared to normal ones. SW1736, $8505 \mathrm{c}$ and Nthy-ori 3-1 were subjected to NanoString platform miRNAs evaluation. (A) A total of 36 and 49 miRNAs turned out to be upregulated in SW1736 and 8505c, respectively, compared to Nthy-ori 3-1. Twenty-eight miRNAs shared a common upregulation in both cell lines. (B) A total of 29 and 47 miRNAs turned out to be downregulated in SW1736 and 8505c, respectively, compared to Nthy-ori 3-1. Seven miRNAs shared a common downregulation in both cell lines.

rating a possible STAT3-dependent cascade alteration. We next evaluated if the ultimate targets of this signaling cascade were upregulated after JQ1 treatment. Both SW1736 and $8505 \mathrm{c}$ exhibited a striking increase in both p21 and p27 protein levels after $5 \mu \mathrm{M}$ JQ1 treatment (Fig. 3A and B).

Differentially expressed miRNAs between ATC and normal thyroid tissue. Finally, to identify chief miRNAs differen-
Table II. Altered miRNAs in ATC cells compared to nontumorigenic ones.

miRNAs commonly dysregulated in ATC cell lines

\begin{tabular}{ll}
\hline Upregulated & Downregulated \\
\hline hsa-miR-1 & hsa-miR-1234 \\
hsa-miR-100-5p & hsa-miR-1268a \\
hsa-miR-1226-3p & hsa-miR-127-3p \\
hsa-miR-1257 & hsa-miR-193b-3p \\
hsa-miR-125b-5p & hsa-miR-34a-5p \\
hsa-miR-139-3p & hsa-miR-4488 \\
hsa-miR-146a-5p & hsa-miR-4516 \\
hsa-miR-183-5p & \\
hsa-miR-221-3p & \\
hsa-miR-29b-3p & \\
hsa-miR-302f & \\
hsa-miR-31-5p & \\
hsa-miR-335-5p & \\
hsa-miR-337-3p & \\
hsa-miR-337-5p & \\
hsa-miR-33a-5p & \\
hsa-miR-412 & \\
hsa-miR-4286 & \\
hsa-miR-450a-5p & \\
hsa-miR-502-5p & \\
hsa-miR-504 & \\
hsa-miR-512-3p & \\
hsa-miR-512-5p & \\
hsa-miR-548a-5p & \\
hsa-miR-548am-3p & \\
hsa-miR-548d-3p & \\
hsa-miR-7-5p & \\
hsa-miR-936 & \\
\hline
\end{tabular}

tially expressed between ATC cells and non-tumorigenic ones, SW1736, 8505c and Nthy-ori 3-1 RNAs were run on the NanoString platform. Considering the 812 miRNAs analyzed on the NanoString platform, 65 and 96 miRNAs turned out to be dysregulated in SW1736 and 8505c cells, respectively, when compared to Nthy-ori 3-1. Among them, miR-146a-5p and miR-221-3p were overexpressed in the two ATC cell lines confirming data already enlisted in literature reviews $(21,24)$. Thirty-five miRNAs exhibited a common dysregulation in the two ATC cell lines when compared to Nthy-ori 3-1 (Fig. 5 and Table II). Several of these have been already described as upregulated in thyroid cancer $(24,25)$ and miR-221/222 have undoubtedly been correlated to cancer aggressiveness (11). Crosschecking Tables I and II, we could highlight that three miRNAs (miR-4516, miR-1234 and miR-4488) exhibited aberrant behavior: they were downregulated in ATC cells when compared to Nthy-ori 3-1 and turned out to be upregulated in ATC after both 48 and $72 \mathrm{~h}$ of JQ1 treatment. 


\section{Discussion}

Recently, the investigation of BET inhibitor-derived miRNA regulation has gained the attention of researchers. Hitherto, only focused studies, aimed to investigate the modulation of one miRNA or one miRNA subfamily after BET inhibition, are available (26). Based on this, our data, for the first time, explored the link between JQ1 and global miRNA expression. In thyroid cancer, miRNA dysregulation has been reported to contribute to tumor progression (27). ATC cells treated with $5 \mu \mathrm{M}$ JQ1 exhibited substantial miRNA dysregulation at both 48 and $72 \mathrm{~h}$ of exposure. We identified several miRNAs substantially upregulated after sustained JQ1 exposure in ATC cells. Some of these miRNAs have already been associated to the inhibition of proliferation, apoptosis induction and have been correlated with survival in different solid tumor models $(28,29)$.

The signal transducer and activator of transcription STAT3 is a transcription factor that gets phosphorylated upon activation, then relocates into the nucleus and activates target genes (30). This protein plays an important role in cellular transformation and tumorigenesis and is constitutively activated in approximately $70 \%$ of solid and hematological cancers $(31,32)$. Increased expression and activation of STAT3 has been described in thyroid cancer tissues and inhibition of the STAT3 signaling pathway has been considered as a novel therapeutic approach to treat human cancers that harbor aberrantly active STAT3 (18). Noteworthy, several miRNAs included in our analysis turned out to be associated to STAT3 signaling pathways, miR-4516 being the most characterized $(17,33)$. STAT3 is known to positively regulate $S P K 2$ gene expression, which in turn mediates the ubiquitination of $\mathrm{p} 21$ and 227 (34). SKP2 is an F-box protein belonging to the SCF E3 ubiquitin ligase complex; it is a known oncogene regulating cellular proliferation, cancer progression and metastasis by inducing the degradation of the cyclin-dependent kinase (Cdk) inhibitors $\mathrm{p} 21^{\mathrm{Cip} 1}, \mathrm{p} 27^{\mathrm{Kip} 1}$ and p57 (21). However, upstream regulators of SKP2 in the progression of human cancer are not yet thoroughly known. Many genes and regulatory pathways have been discovered (STAT3, BCR-ABL, PI3K/AKT, ERK, PPAR $\gamma$, PTEN and mTOR) making SKP2 regulation a complex issue in cancer research (22). Nevertheless, several studies have demonstrated a direct and solid link between STAT3 phosphorylation and the SKP2/p27 axis. JQ1 treatment undeniably produced a decrease in SKP2 expression correlated to an increase in p21 and p27 protein levels, in both ATC cell lines. STAT3 phosphorylation was subjected to a strong decrease in cell line $8505 \mathrm{c}$, while it was undetectable in SW1736 cells, probably due to a diverse site of phosphorylation from the one detected in our analysis.

In conclusion, our results highlighted that a JQ1-regulated miRNA impaired STAT3 transduction pathways leading to antineoplastic activities in ATC cell lines. Both ATC cell lines displayed an upregulation of some miRNAs enlisted in multiple ATC literature reviews (i.e., miR-146a and miR-221). An interesting event could be disclosed comparing JQ1-regulated and ATC-linked miRNAs: three miRNAs (miR-4516, miR-1234, miR-4488) were determined to be downregulated in both ATC cell lines when compared to Nthy-ori 3-1 and, then, turned out to be upregulated by JQ1 treatment, after both 48 and $72 \mathrm{~h}$ of exposure. In brief, these data revealed the antineoplastic activity of JQ1 in thyroid cancer cells.

\section{Acknowledgements}

We thank Dr Emanuele Cettul and Dr Luciana Gualdi from Pharmadiagen S.r.l. (Pordenone, Italy) for the miRNome profiling. This study was supported by a grant to GD from Ministero degli Affari Esteri of Italy (Progetti grande rilevanza 2016, project no. PGR02954) and from MIUR (PRIN 2015, project no. 2015HPMLFY-011).

\section{References}

1. Gu L and Sun W: MiR-539 inhibits thyroid cancer cell migration and invasion by directly targeting CARMA1. Biochem Biophys Res Commun 464: 1128-1133, 2015.

2. Gao X, Wu X, Zhang X, Hua W, Zhang Y, Maimaiti Y, Gao Z and Zhang Y: Inhibition of BRD4 suppresses tumor growth and enhances iodine uptake in thyroid cancer. Biochem Biophys Res Commun 469: 679-685, 2016.

3. Mio C, Lavarone E, Conzatti K, Baldan F, Toffoletto B, Puppin C, Filetti S, Durante C, Russo D, Orlacchio A, et al: MCM5 as a target of BET inhibitors in thyroid cancer cells. Endocr Relat Cancer 23: 335-347, 2016.

4. Nicodeme E, Jeffrey KL, Schaefer U, Beinke S, Dewell S, Chung CW, Chandwani R, Marazzi I, Wilson P, Coste H, et al: Suppression of inflammation by a synthetic histone mimic. Nature 468: 1119-1123, 2010.

5. Fu LL, Tian M, Li X, Li JJ, Huang J, Ouyang L, Zhang Y and Liu B: Inhibition of BET bromodomains as a therapeutic strategy for cancer drug discovery. Oncotarget 6: 5501-5516, 2015.

6. Cheng Z, Gong Y, Ma Y, Lu K, Lu X, Pierce LA, Thompson RC, Muller S, Knapp S and Wang J: Inhibition of BET bromodomain targets genetically diverse glioblastoma. Clin Cancer Res 19: 1748-1759, 2013.

7. Sahni JM, Gayle SS, Bonk KLW, Vite LC, Yori JL, Webb B, Ramos EK, Seachrist DD, Landis MD, Chang JC, et al: Bromodomain and extraterminal protein inhibition blocks growth of triple-negative breast cancers through the suppression of aurora kinases. J Biol Chem 291: 23756-23768, 2016.

8. Dawson MA, Prinjha RK, Dittmann A, Giotopoulos G, Bantscheff M, Chan WI, Robson SC, Chung CW, Hopf C, Savitski MM, et al: Inhibition of BET recruitment to chromatin as an effective treatment for MLL-fusion leukaemia. Nature 478: 529-533, 2011.

9. Filippakopoulos P, Qi J, Picaud S, Shen Y, Smith WB, Fedorov O, Morse EM, Keates T, Hickman TT, Felletar I, et al: Selective inhibition of BET bromodomains. Nature 468: 1067-1073, 2010.

10. Li L, Lv B, Chen B, Guan M, Sun Y, Li H, Zhang B, Ding C, He S and Zeng Q: Inhibition of miR-146b expression increases radioiodine-sensitivity in poorly differential thyroid carcinoma via positively regulating NIS expression. Biochem Biophys Res Commun 462: 314-321, 2015.

11. Fuziwara CS and Kimura ET: MicroRNA Deregulation in anaplastic thyroid cancer biology. Int J Endocrinol 2014: 743450, 2014.

12. Borrelli N, Denaro M, Ugolini C, Poma AM, Miccoli M, Vitti P, Miccoli P and Basolo F: miRNA expression profiling of 'noninvasive follicular thyroid neoplasms with papillarylike nuclear features' compared with adenomas and infiltrative follicular variants of papillary thyroid carcinomas. Mod Pathol 30: 39-51, 2017.

13. Pilli T, Prasad KV, Jayarama S, Pacini F and Prabhakar BS: Potential utility and limitations of thyroid cancer cell lines as models for studying thyroid cancer. Thyroid 19: 1333-1342, 2009.

14. Schweppe RE, Klopper JP, Korch C, Pugazhenthi U, Benezra M, Knauf JA, Fagin JA, Marlow LA, Copland JA, Smallridge RC, et al: Deoxyribonucleic acid profiling analysis of 40 human thyroid cancer cell lines reveals cross-contamination resulting in cell line redundancy and misidentification. J Clin Endocrinol Metab 93: 4331-4341, 2008.

15. Passon N, Gerometta A, Puppin C, Lavarone E, Puglisi F, Tell G, Di Loreto $C$ and Damante G: Expression of Dicer and Drosha in triple-negative breast cancer. J Clin Pathol 65: 320-326, 2012. 
16. Gatfield D, Le Martelot G, Vejnar CE, Gerlach D, Schaad O, Fleury-Olela F, Ruskeepää AL, Oresic M, Esau CC,Zdobnov EM, et al: Integration of microRNA miR-122 in hepatic circadian gene expression. Genes Dev 23: 1313-1326, 2009.

17. Chowdhari S and Saini N: hsa-miR-4516 mediated downregulation of STAT3/CDK6/UBE2N plays a role in PUVA induced apoptosis in keratinocytes. J Cell Physiol 229: 1630-1638, 2014.

18. Dong W, Cui J, Tian X, He L, Wang Z, Zhang P and Zhang H: Aberrant sonic hedgehog signaling pathway and STAT3 activation in papillary thyroid cancer. Int J Clin Exp Med 7: 1786-1793, 2014.

19. Yan LI,Li LI,Li Q, Di W, Shen W, Zhang Land Guo H: Expression of signal transducer and activator of transcription 3 and its phosphorylated form is significantly upregulated in patients with papillary thyroid cancer. Exp Ther Med 9: 2195-2201, 2015.

20. Zhang J, Gill A, Atmore B, Johns A, Delbridge L, Lai R and McMullen T: Upregulation of the signal transducers and activators of transcription 3 (STAT3) pathway in lymphatic metastases of papillary thyroid cancer. Int J Clin Exp Pathol 4 : 356-362, 2011.

21. Lee J-J, Lee J-S, Cui MN, Yun HH, Kim HY, Lee SH and Lee J-H: BIS targeting induces cellular senescence through the regulation of 14-3-3 zeta/STAT3/SKP2/p27 in glioblastoma cells. Cell Death Dis 5: e1537, 2014.

22. Bochis OV, Irimie A, Pichler M and Berindan-Neagoe I: The role of Skp2 and its substrate CDKN1B (p27) in colorectal cancer. J Gastrointestin Liver Dis 24: 225-234, 2015.

23. Sos ML, Levin RS, Gordan JD, Oses-Prieto JA, Webber JT, Salt M, Hann B, Burlingame AL, McCormick F, Bandyopadhyay S, et al: Oncogene mimicry as a mechanism of primary resistance to BRAF inhibitors. Cell Reports 8: 1037-1048, 2014

24. Wójcicka A, Kolanowska M and Jażdżewski K: Mechanisms in endocrinology: MicroRNA in diagnostics and therapy of thyroid cancer. Eur J Endocrinol 174: R89-R98, 2016.
25. Leonardi GC, Candido S, Carbone M, Colaianni V, Garozzo SF, Cinà D and Libra M: microRNAs and thyroid cancer: Biological and clinical significance (Review). Int J Mol Med 30: 991-999, 2012.

26. Xu Z, Sharp PP, Yao Y, Segal D, Ang CH, Khaw SL, Aubrey BJ, Gong J, Kelly GL, Herold MJ, et al: BET inhibition represses miR17-92 to drive BIM-initiated apoptosis of normal and transformed hematopoietic cells. Leukemia 30: 1531-1541, 2016.

27. Forte S, La Rosa C, Pecce V, Rosignolo F and Memeo L: The role of microRNAs in thyroid carcinomas. Anticancer Res 35: 2037-2047, 2015.

28. Xu X, Wu J, Li S, Hu Z, Xu X, Zhu Y, Liang Z, Wang X, Lin Y, Mao Y, et al: Downregulation of microRNA-182-5p contributes to renal cell carcinoma proliferation via activating the AKT/ FOXO3a signaling pathway. Mol Cancer 13: 109, 2014.

29. Liu N, Cui R-X, Sun Y, Guo R, Mao YP, Tang LL, Jiang W, Liu X, Cheng YK, He QM, et al: A four-miRNA signature identified from genome-wide serum miRNA profiling predicts survival in patients with nasopharyngeal carcinoma. Int J Cancer 134: 1359-1368, 2014.

30. Sosonkina N, Starenki D and Park J-I: The Role of STAT3 in Thyroid Cancer. Cancers (Basel) 6: 526-544, 2014.

31. Frank DA: STAT3 as a central mediator of neoplastic cellular transformation. Cancer Lett 251: 199-210, 2007.

32. Sansone P and Bromberg J: Targeting the interleukin-6/Jak/stat pathway in human malignancies. J Clin Oncol 30: 1005-1014, 2012.

33. Chowdhari S and Saini N: Gene expression profiling reveals the role of RIG1 like receptor signaling in p53 dependent apoptosis induced by PUVA in keratinocytes. Cell Signal 28: 25-33, 2016.

34. Lin H-P, Lin C-Y, Huo C, Hsiao PH, Su LC, Jiang SS, Chan TM, Chang CH, Chen LT, Kung HJ, et al: Caffeic acid phenethyl ester induced cell cycle arrest and growth inhibition in androgenindependent prostate cancer cells via regulation of Skp2, p53, p21 $1^{\mathrm{ipl}}$ and $\mathrm{p} 27^{\mathrm{Kipl}}$. Oncotarget 6: 6684-6707, 2015. 